\title{
'||||||||||||||||||||||||||||||||||||||||||||||||||||||||||||||||||||||.
}

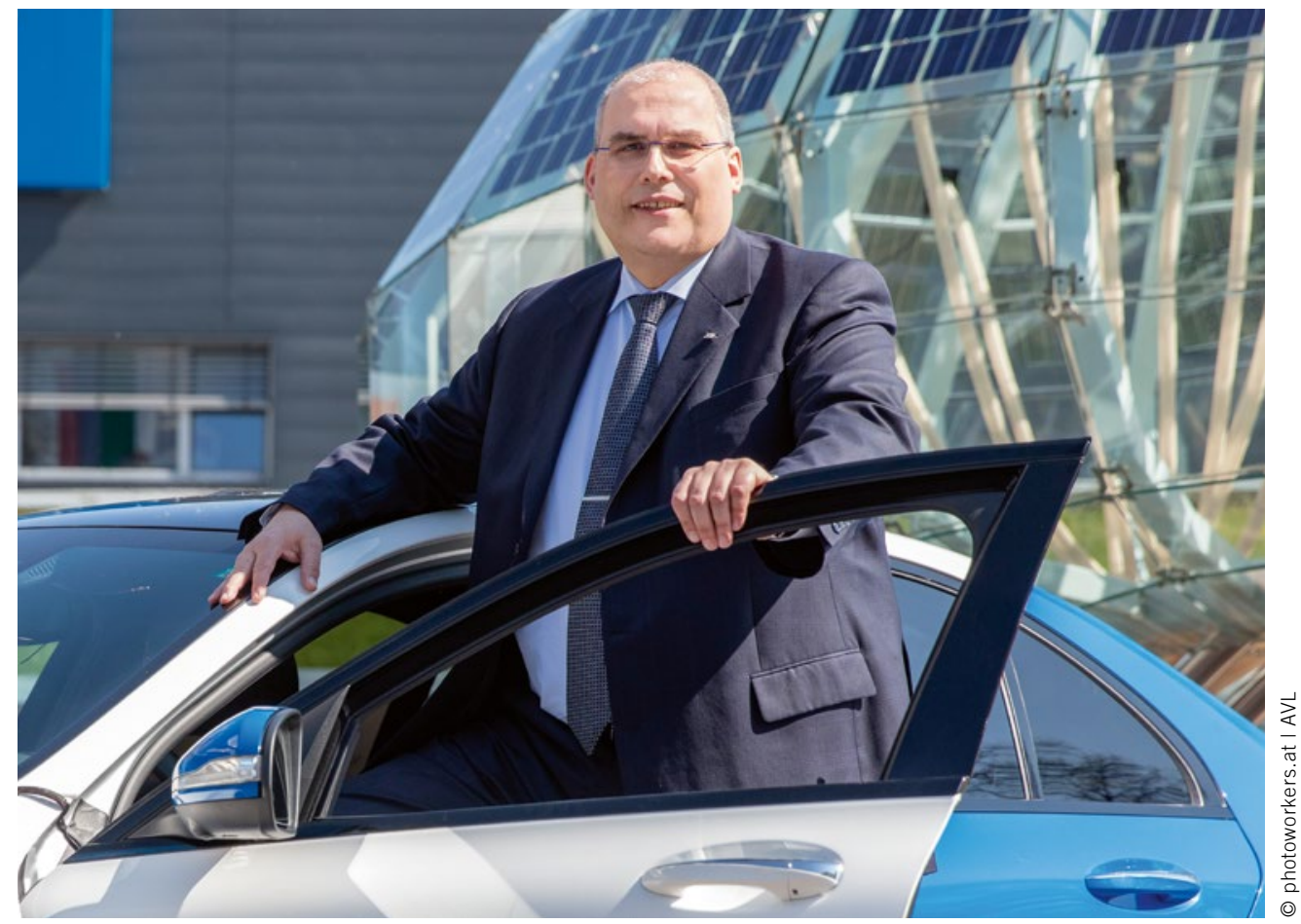

Prof. Dr. Uwe Dieter Grebe Executive Vice President, AVL List GmbH, Member of the ATZ Advisory Board

\section{Vehicle Attributes in a New World}

The market success of vehicles depends on the extent to which vehicle attributes are matching customer requirements and on affordability. Differing legislations around the globe and the threat of locally imposed bans on driving are forcing automobile manufacturers to offer a wide variety of propulsion systems with increasing levels of electrification. Advanced driver assistance systems and autonomous driving are transforming the market and play a crucial role in emerging mobility concepts.

In this new and evolving world, cleverly designed modular systems incorporating vehicle platforms and drive systems enable cost-optimised model configurations. Functional integration determines the vehicle character and is fundamental to its success. In this respect, complete vehicle simulation with functionally linked vehicle component models is becoming increasingly important. It makes the complex interrelationships transparent and permits the assessment of different vehicle architectures in view of their effect on customers early on in the conceptual stage. Here at AVL, we couple vehicle simulation with the driving simulator and make attribute-engineering solutions a "tangible" experience.

The vehicle sub systems' operating strategies are formative for the vehicle attributes. The complete vehicle simulation in real-time during driving operation enables mechanical, thermal, electrical and hydraulic power flow optimisation while achieving the best possible driving experience. For this specific purpose, AVL has developed the "Energy Broker" methodology, which makes all the decisions relating to vehicle energy supply, distribution and storage. Connectivity between vehicles and with the environment opens up further opportunities to optimise vehicle attributes situationally. Traffic and route information can also be incorporated to individualise the vehicles.

Development processes for autonomous vehicles require the integration of new methods. Real-world tests and computational simulations are merged. For this purpose, we established a special test and simulation centre in Graz, which opened in the middle of 2017. Autonomous vehicles, also operating on our own test track and on special highway sections approved by the Austrian Federal Government, deliver data on a continuous basis. The evaluation of the driving situations is based on AVLDrive ADAS, a tool that carries out an objective assessment of the passengers' perception of safety.

I am confident that in future the individualisation of driving attributes will also be available to the end-customer. In the near future, customers will be using shared vehicles which set their driving attributes according to a specific passenger profile. In a digitised world, this opens up new opportunities for vehicle attribute optimisation, which will contribute significantly towards success with end-customers. 